\title{
Rapid gastric ulceration in a modified two-way shuttlebox*
}

\author{
LEONARD W. HAMILTON and FLOYD A. KATSKE $\dagger$ \\ Rutgers University, New Brunswick, New Jersey 08903
}

\begin{abstract}
A modification of the standard two-way shuttlebox procedure resulted in extensive gastric erosions within a 1-h testing period. The results suggest that the uncertainty regarding the onset and termination of shock in combination with the spatial conflict induced by this situation lead to severe psychological stress.
\end{abstract}

The investigation of the determinants of gastric ulceration has been of considerable interest, not only because of the possibility of solving a pervasive medical problem, but also because of its implications for certain aspects of learning theories. Most of the experimental paradigms that have been used in the investigation of ulceration have involved an aversive stimulus presented according to some predetermined schedule.

Two major hypotheses have been advanced to account for the development of ulceration: the control hypothesis and the conflict hypothesis. The basic assumption of the control hypothesis is that the ability to exert control over the duration (or frequency of presentation) of the aversive stimuli results in an attenuation of the aversive properties of the stimuli. An example of experimental data supporting this viewpoint is that of Moot, Cebulla, and Crabtree (1970), who trained rats to work for food on a variable interval (VI) schedule and then paired each food reinforcement with electrical shock. Testing under these conditions was continued for three 20 -h sessions. Following behavioral testing, it was found that rats that were able to control the duration of the electrical shock developed significantly fewer ulcers than did yoked controls that experienced the same frequency and duration of shock but had no control over it.

The conflict hypothesis suggests that a major determinant of gastric ulceration is the degree of psychological conflict engendered by the introduction of the aversive stimuli. In an experiment designed to test the importance of psychological conflict, Weiss (1971a) tested rats for 48 consecutive hours in an escape-avoidance situation in which a brief pulse of shock accompanied each escape or avoidance response. Rats in the "punished" condition developed significantly more ulcers than did yoked controls, even though they had some degree of instrumental control over the situation.

*This research was supported in part by funds from the Rutgers University Research Council. Reprint requests should be mailed to Leonard W. Hamilton, Department of Psychology, Rutgers University, New Brunswick, New Jersey 08903.

tSome of the data presented in this report were collected by the second author in partial fulfillment of the requirements of the Henry Rutgers research program.
It should be noted, for both of these examples, that the Ss were exposed to the aversive situation for extended periods of time. The present investigation examined the efficacy of introducing additional conflict components into a classic conflict situation-the two-compartment shuttlebox.

\section{METHOD}

\section{Subjects}

For Experiment 1, the Ss were 16 adult male albino rats of the Sprague-Dawley strain obtained from Carwoth (New York). For Experiment 2, the Ss were 16 adult male hooded rats obtained from Blue Spruce Farms (New York). Rats in both experiments were deprived of food during the $48-\mathrm{h}$ period preceding testing, but water was available ad lib.

\section{Apparatus}

The shuttlebox was constructed of transparent Plexiglas. Each compartment of the chamber was $20 \times 35 \times 30 \mathrm{~cm}$ high with $0.5-\mathrm{cm}$ stainless steel grids spaced $2 \mathrm{~cm}$ apart for the floor. Constant-current electric shock $(0.8 \mathrm{~mA})$ could be delivered through the grid floor from a shock source modified after that described by Brown, Reus, and Webb (1961). There was no partition between the two compartments.

\section{Procedure}

The basic rationale in the design of the experimental procedures was to increase the conflict which normally is inherent in the two-way escape avoidance procedure. As a result (apparently) of a combination of apparatus dimensions, program parameters, and shock source, the apparatus used in the present study induces a great deal of conflict and poor avoidance acquisition. For example, the normal rats used by Hamilton (1970) showed less than 20\% avoidance responses after six 20-trial training sessions. Because of the high degree of conflict that was present with a signaled avoidance contingency in effect, this situation was thought to be ideal for producing an ulcerogenic procedure.

Two modifications of the apparatus were introduced to increase the conflict engendered by the situation: (a) The partition which normally separated the two chambers was removed to make the cues associated with location less obvious, and (b) the CS light which would normally provide the opportunity for an avoidance response was eliminated.

In addition to the two physical modifications noted above, variations in the automatically programmed shock presentation defined the different testing conditions as follows: Condition $\mathrm{CON}$-control procedure in which shock was never presented; Condition FI/VI-continuous escapable electric shock 


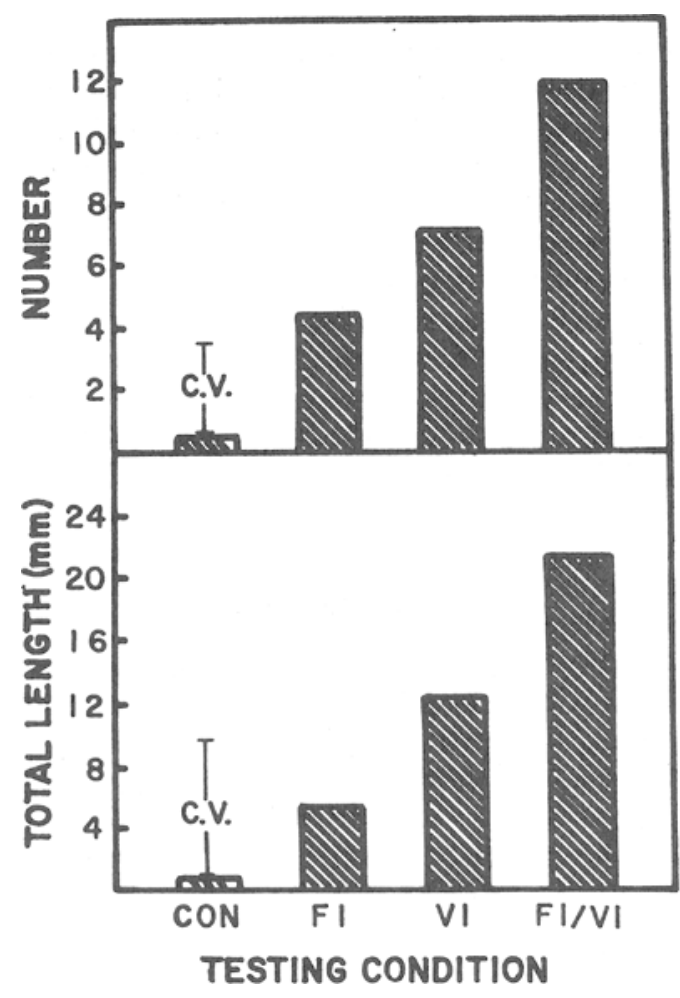

Fig. 1. Average number and total length of gastric erosions produced by the four testing conditions (see text for explanation of conditions). The critical value (c.v.) obtained for the Fisher's test of least significant differences is indicated. Differences between means of this value or greater are significant at the .05 level.

was alternated from one compartment to the other according to a 40-sec fixed-interval (FI) schedule, and discrete 2-sec inescapable shocks were delivered in the nonshock compartment on a variable-interval (VI) 1-min schedule; Condition FI-only the escapable FI shocks were delivered; and Condition VI-only the inescapable VI shocks were delivered (to both chambers simultaneously).

Experiment 1 was designed primarily to test the efficacy of the FI/VI condition. Accordingly, 5 of the rats were assigned to the CON condition and the remaining 11 rats were assigned to the FI/VI condition. Experiment 2 tested the efficacy of the separate FI and VI components of the schedule by assigning four rats to each of the four testing conditions.

Following the experimental treatment, all rats were examined for evidence of ulceration. Each animal received an overdose of Equi-Thesin (Jensen-Salsbery), following which the stomach was removed and rinsed clean. The stomach was then opened by an incision from the pyloris to the esophagus, along the greater curvature, and turned out for examination. The extent of pathology (which was invariably restricted to the glandular portion of the stomach) was assessed by quantifying the size and frequency of blood-filled gastric erosions with the aid of a dissecting microscope.

\section{RESULTS}

\section{Experiment 1}

The results of Experiment 1 revealed that Condition FI/VI was very effective in producing gastric pathology. All of these rats developed obvious pathology, ranging in severity from several small erosions in a reddish-colored, irritated mucosa to extensive blood-filled erosions. By contrast, no pathology was observed in the stomachs of rats in the control condition, nor did the stomach mucosa have the reddish appearance that was typical for the control rats. This difference in frequency of ulceration is, of course, highly significant $\left(\chi^{2}=9.78, \mathrm{df}=1, \mathrm{p}<.01\right)$.

\section{Experiment 2}

The results of Experiment 2, summarized in Fig. 1, provide a more detailed account of the conditions which lead to ulceration. An analysis of variance was performed on the number of lesions observed and the four groups were found to differ significantly ( $F=$ 25.50 , df $=3 / 12, p<.001)$. Subsequent two-sample comparisons were made using Fisher's test of least significant differences (Winer, 1971). Application of the resulting critical value of $2.96(p=.05)$ to the group means revealed the following relationships of the four groups: $\mathrm{CON}<\mathrm{FI}=\mathrm{VI}<\mathrm{FI} / \mathrm{VI}$.

It is clear that either the presentation of escapable shocks according to an FI schedule or the presentation of inescapable shocks according to a VI schedule can lead to a significant degree of ulceration. The combination of the two schedules was, however, considerably more effective than either condition presented singly. A similar relationship was observed for the total length of ulcers $(F=10.10$, df $=3 / 12$, $\mathrm{p}<.005$ ), except that Condition FI did not differ significantly from the control condition.

In addition to pointing out some of the relevant variables in this procedure, Experiments 1 and 2 show that the gastric ulceration is produced in at least two strains of rats. Although direct quantitative comparisons were not made, the extent of gastric pathology produced by Condition FI/VI appeared to be comparable for both hooded and albino rats.

\section{DISCUSSION}

The procedures outlined in this study were found to be extremely effective in producing gastric pathology. In contrast to the typical procedures (e.g., Moot et al, 1970; Weiss, 1971a, b), which utilized test sessions lasting many hours or multiple test sessions extending across days, the present procedures produced severe gastric pathology during a single 1-h test session. The rapidity and severity of these effects suggest that the combination of experimental parameters that were used resulted in severe psychological stress. An examination of the various components of this ulcerogenic situation may point out the nature of this psychological stress.

Condition FI was essentially a modification of the standard two-way escape-avoidance procedure. In the two-way active avoidance procedure, conflict is 
introduced by the requirement that the $S$ return to the compartment from which it recently escaped or avoided shock. In the present situation, there was no warning signal; each response was, therefore, either an escape response or a punished intertrial crossing, and the time of shock onset was uncertain (except for temporal cures). A further element of conflict may have been introduced by the removal of the center partition, making it more difficult to discriminate the boundary between the two compartments. The importance of conflict in ulcerogenesis is emphasized by the fact that gastric pathology developed rapidly, even though the rats had instrumental control over the duration of the shock.

The VI component, presented singly, was also effective in producing ulcers. Presumably, the basis for this effect was the uncertainty of the time of shock onset and the lack of instrumental control over the shock duration.

The combination of these two components (Condition FI/VI) greatly exaggerated the effectiveness in producing gastric erosions. The most likely cause of this increased effectiveness is that the combination of the two schedules makes the result of a response to shock uncertain-if the shock onset is the result of the FI program, jumping to the other chamber results in escape; if the shock onset is the result of the VI program, a response will place the rat in the compartment that is electrified throughout the trial, forcing a retreat. Thus, the combination of uncertainty concerning the time of shock onset and the result of a response to shock leaves the organism in a more or less hopeless situation.
In conclusion, we believe that the effectiveness of this situation in producing pathology is primarily attributable to the high degree of conflict rather than to a lack of instrumental control, although the latter is certainly effective to a certain extent. Furthermore, the unusually rapid production of gastric pathology suggests that conflict involving spatial cues, in the rat at least, may be more salient than that induced by instrumental schedules alone. These procedures may be useful both in terms of their implications for theories of ulcerogenesis and as an efficient tool for the investigation of therapeutic measures.

\section{REFERENCES}

Brown, C. C., Reus, J. F., \& Webb, G. A new constant current stimulation circuit. Proceedings of the International Conference of Medical Electronics, 1961, p. 200.

Hamilton, L. W. Behavioral effects of unilateral and bilateral septal lesions in rats. Physiology \& Behavior, 1970, 5, 855-859.

Moot, S. A., Cebulla, R. P., \& Crabtree, J. M. Instrumental control and ulceration in rats. Journal of Comparative \& Physiological Psychology, 1970, 71, 405-410.

Weiss, J. M. Effects of coping behavior in different warning signal conditions on stress pathology in rats. Journal of Comparative \& Physiological Psychology, 1971a, 77, 1-13.

Weiss, J. M. Effects of punishing the coping response (conflict) on stress pathology in rats. Journal of Comparative \& Physiological Psychology, 1971b, 77, 14-21.

Winer, B. J. Statistical principles in experimental design. New York: McGraw-Hill, 1971.

(Received for publication December 12, 1972; revision accepted January 29, 1973.) 